\title{
State Assignment Selection for FPGAs and CPLDs
}

\author{
Hichem BELHADJ, Aziz FORTAS and Gabrièle SAUCIER \\ Laboratoire CSI-Institut National Polytechnique de Grenoble \\ 46, Avenue Félix Viallet 38031 Grenoble Cedex, FRANCE \\ Tel. (33) 765748 20, Fax.(33)76 5034 21, Hichem.Belhadj@imag.fr
}

\begin{abstract}
This paper deals with the selection of state assignment options for finite state machines implemented in various targets. This choice depends on the intrinsic controller complexity, the partitioning strategy, the area/delay constraints and the target technologies. Industrial controllers as well as a sub-set of MCNC benchmarks have been synthesized and analyzed after place and route to define the selection strategy.
\end{abstract}

\section{Keywords}

Finite state machine synthesis, State assignment, FPGAs and CPLDs.

\section{INTRODUCTION}

The optimization of finite state machine synthesis depends primarily on the state assignment and secondly on the minimization of the next state and output logic. The problem has been extensively addressed in the past. The optimization criteria being either the number of product terms or the number of literals of the next state or output logic. An important parameter is the number of memory elements used to store the internal state. In some cases, a minimal number is an important goal ; in some others a larger number of memory elements is quite acceptable or suitable. All these options have to be analyzed with regards to target technology and in the past a lot of attention has been given to PLA implementation [(De Micheli, 1983), (Villa, 1986), (Paulin, 1989), (Amman, 1988)] and to standard cells or gate arrays [(Lin, 1989), (Devadas, 1987)] and only a few works have considered the programmable devices [(Golson, 1993), (Boriello, 1993)]. This paper considers 3 types of state assignments : the well known one hot encoding, the compact encoding aiming at reducing both the number of product terms and the literal count of the next state and output functions, and the linear encodings tending to identify paths in the finite state machine state groups and to encode the states of these paths with successive linear code elements.

The goal is to analyze which state assignment is the "best" for a given constraint (area or speed), for a controller of a given complexity, and for a given target technology. The target technologies are namely FPGAs and PLDs, and experiments have been done on technologies where the prediction of the critical path is easy and accurate namely Actel, Xilinx, QuickLogic, Crospoint, MACH, PAL and for comparison for standard cell library.

This paper is organised as follows. Section 1 presents the different encoding technics used in the experimentation. The complexity of a finite state machine is introduced in section 2 . The significant differences between several technological targets and their effect on the selection of the state assignment is detailed in section 3. Section 4 presents the experimentation scripts and analyzes the obtained results. It also presents the state assignment selection strategy. 


\section{THE DIFFERENT STATE ASSIGNMENTS}

\section{I.1. One Hot encoding}

This technique uses one register for each state, i.e for each state only one of $n$ internal variables has the value 1 , where $\mathrm{n}$ is the number of states.

\section{I.2. Compact state assignment}

The compact state assignment uses the minimal number of memory elements and tends to minimize both the number of product terms and the literal count in the next state and output functions. It uses a sophisticated and powerful method tending to reduce the complexity of these equations. This method is the extension of the partition pair theory of Stearn and Hartmanis (Hartmanis, 1966) and defines sub-sets of states to be placed on faces of the hypercube to reach this reduced dependency. In a second step, an efficient face embedding technique yields the final state assignment (Duff, 1991). Extensive practical experiments on about a hundred of FSMs have shown after place and route significant benefits in both area and critical path over JEDI (Newton, 1989) and NOVA (Villa, 1989).

\section{3. Path encoding}

This state assignment is suitable when the state graph exhibits long paths. In this case, a preprocessing phase partitions the state graph inte paths and branching arcs and then applies linear codes on the paths.

\section{I.3.1 Partitioning strategy}

Some basic definitions need to be introduced before the partitioning strategy can be explained. * The minterm associated with the binary code $\left(\mathrm{c}_{\mathrm{n}} \ldots \mathrm{c}_{1} \mathrm{c}_{0}\right)$ assigned to a node of the control flow-graph is $\tilde{y}_{n} \ldots \tilde{y}_{0}$, where $\tilde{y}_{i}=y_{i}\left(\overline{y_{i}}\right)$ if $c_{i}=1(0)$. For instance, if the code of the node $S$ is "1010" then the minterm associated with $S$ is $\overline{y_{0}}$ y1 $\overline{y_{2}}$ y3.

* The Boolean expression produced by an arc a, denoted by $\mathrm{SF}_{\mathrm{a}}$, is the product of the minterm associated with the origin node multiplied by the input firing condition, or input predicate, labelling the arc a. An example of this is to assume that an arc a issued from the state $S$ is labelled by the input predicate $(e+f)$. Then the Boolean expression produced by this transition (or arc) is $S_{\mathbf{a}}=(e+f)$. ( $\left.\overline{y_{0}} \mathrm{y}_{1} \overline{y_{2}} y_{3}\right)$.

* The next state equation is defined as $\mathrm{Y}_{\mathrm{t}+1}=\sum_{\mathrm{a} \in \mathrm{A}} \mathrm{SF}_{\mathrm{a}}$ ( $\mathrm{yt}_{\mathrm{t}}$, Input), where $\mathrm{A}$ is the set of arcs.

Let $\mathrm{A}$ denote the set of arcs of the state graph. If $\mathrm{SF}_{\mathrm{a}}$ is the Boolean expression produced by an $\operatorname{arc} \mathrm{a}$, and $\mathrm{L}\left(\mathrm{SF}_{\mathrm{a}}\right)$ represents the literal count of $\mathrm{F}$; then a partitioning strategy, consisting of splitting $A$ into sub-sets $A 1, \ldots, A n$, is cost effective if $\sum_{a \in A} L\left(S_{a}\right)<L\left(\sum_{i} S F_{A_{i}}\right)$.

It has been stated in [BGLS93], that one of the best strategies consists of spliting the set of $\operatorname{arcs} \mathrm{A}$ into three sub-sets $\mathrm{A} 1, \mathrm{~A} 2$ and $\mathrm{A} 3$, where

- A1 is the set of arcs whose origin node has only one successor node and whose destination node is the destination of only one arc.

- $\quad \mathrm{A} 2$ is the set of arcs whose origin node has several successor nodes.

- $\quad \mathrm{A} 3$ is the set of arcs whose the destination nodes have several predecessor nodes. 
In the following only path encoding is addressed. In a path, each arc maps bijectively a present state code to a next state code. A path encoding consists in defining a path function fP such that, for each couple (present state $S_{i}$, next state $\left.S_{i+1}\right), \operatorname{Code}\left(S_{i+1}\right)=f P\left(\operatorname{Code}\left(S_{i}\right)\right)$.

The linear code assignment consists of assigning the nodes of the sub set A1 to linear codes and the arcs to faces of the hypercube; this leads to the study of the cubical compactness .

\section{I.3. 2 Cubical compactness of a path encoding}

The path encoding is said to be cubical compact if by fixing a predefined code to the initial state $S_{0}$, the following property can be obtained on the hypercube ${ }^{1}$ :

The codes $\left(\mathrm{S}_{0}, \delta\left(\mathrm{S}_{0}\right), \ldots, \delta^{3}\left(\mathrm{~S}_{0}\right)\right)$ define a 2 -cube in the hypercube,

The codes $\left(\mathrm{S}_{0}, \delta\left(\mathrm{S}_{0}\right), \ldots, \delta^{7}\left(\mathrm{~S}_{0}\right)\right)$ define a 3 -cube in the hypercube, and more generally,

The codes $\left(\mathrm{S}_{0}, \delta\left(\mathrm{S}_{0}\right), \ldots, \delta^{2^{\mathrm{n}}-1}\left(\mathrm{~S}_{0}\right)\right)$ define an $\mathrm{n}$-cube in the hypercube.

The cubical compactness plays an important role in the global state assignment and has therefore to be analyzed for the main codes which can be chosen on a path.

\section{3. 3. Study of the different path encodings}

Two commonly used path encodings will be analyzed with respect to the complexity of the logic they produce, estimated by the literal count, and to the cubical compactness property.

\section{A. Radix-2 or sequential code}

The radix-2 code is one of the most commonly used encodings. The path is encoded by assigning to the states $s_{0}, \ldots, s_{n}-1$ sequential binary codes. The code for the state $s_{j}$ is therefore equal to the code for the state $s_{j-1}$ plus one. If $\left(y_{1}, \ldots, y_{k}\right)$ represents the current state then the next state $\left(\mathrm{Y}_{1}, \ldots \mathrm{Y}_{\mathrm{k}}\right)$ is obtained by means of the following formula :

$$
Y_{1}=\overline{y_{1}} \quad \text { and } Y_{i}=y_{i} \cdot \sum_{j=1}^{i-1} \overline{y_{j}}+\overline{y_{i}} \cdot \prod_{j=1}^{i-1} y_{j} \quad \text { for } i=2, \ldots, k
$$

\section{B. Gray code}

Let $c_{m} \ldots c_{1} c_{0}$ denote a code in the Gray code, and let $b_{m} \ldots b_{1} b_{0}$ designate the corresponding binary number, where the subscripts $\mathrm{n}$ and 0 denote the most significant and the least significant digits respectively. Then the ith digit $c_{i}$ can be obtained form the corresponding binary number as follows: $\left.\left.c_{i}=b_{i} \oplus b_{i+1} 0 \leq i<m ;(m=\log 2 n)\right]\right)$ and $c_{m}=b_{m}$, where $\oplus$ denotes the modulo-2 sum. If $\left(\mathrm{y}_{1}, \ldots, \mathrm{y}_{\mathrm{k}}\right)$ represents the current state then the next state $\left(\mathrm{Y}_{1}, \ldots \mathrm{Y}_{\mathrm{k}}\right)$ is obtained by means of the following formula :

$$
\left.Y_{1, k}=\overline{\left(y_{k} \oplus \ldots \oplus y_{2}\right)} ; Y_{i, k}=y_{i} \oplus \overline{\left(y_{k} \oplus \ldots \oplus y_{i}\right)} \cdot y_{i-1} \cdot \prod_{j=1}^{i-2} \overline{y_{j}}\right) \text { for } i=2, \ldots, k-1 \text {, and }
$$

\footnotetext{
${ }^{1}$ The hypercube is represented as the lattice of the parts of $\mathrm{N}$ elements. Each node is labelled by its relative coordinates denoted by $\varepsilon$. $\varepsilon$ is a set of integers $\left(e_{1}, \ldots, e_{n}\right)$. It may be understood as follows: $e_{i} \in \varepsilon$ if and only if the ith bit of the code of that node is equal to 1 . Figure 1 shows three representations of a 3-cube using binary codes, equivalent decimal codes and the lattice of the parts of $\mathrm{N}$ elements. A cube is defined by a pair $\left[\varepsilon_{\min }, \varepsilon_{\max }\right]$ with $\varepsilon_{\max } \geq \varepsilon_{\min }$. For instance $[2,123]$ is a cube of the hypercube of dimension 2. $\varepsilon_{\max }=\varepsilon_{\min }+\varepsilon_{c} ; \varepsilon_{c}$ is called the characteristic set of indices of a cube, and $\left|\varepsilon_{c}\right|$ gives the dimension of the cube. [2, 123] has as characteristic indices $\{13\}$.
}

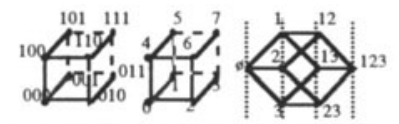

Figure 1: Different representations of a 3-cube 
$\mathrm{Y}_{\mathrm{k}, \mathrm{k}}=\mathrm{y}_{\mathrm{k}} \oplus\left(\left(\mathrm{y}_{\mathrm{k}} \oplus \mathrm{y}_{\mathrm{k}-1}\right) . \prod_{\mathrm{j}=1}^{\mathrm{i}-2} \overline{\mathrm{y}_{\mathrm{j}}}\right)$

Figure 2 illustrates the cubical compactness of the sequential and the Gray codes, and Figure 3 shows the complexity of the derived next-state functions, in terms of literal count, for paths of different lengths. On the other hand, it is easy to verify that the one hot encoding does not verify the cubical compactness property since $\left(\mathrm{S}_{0}, \delta\left(\mathrm{S}_{0}\right), \ldots, \delta^{2^{\mathrm{n}}-1}\left(\mathrm{~S}_{0}\right)\right)$ defines a $\left(2^{\mathrm{n}}-1\right)$-cube.
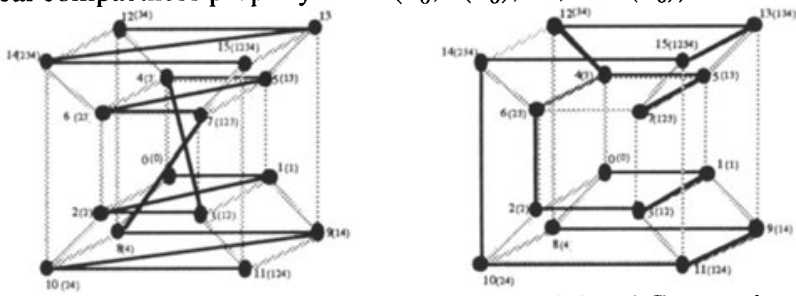

Figure 2 : Cubical compactness of Sequential and Gray codes.

\begin{tabular}{|c|c|c|c|}
\hline Path Lengths & 8 & 16 & 32 \\
\hline Sequential & 11 & 17 & 23 \\
\hline Gray & 12 & 26 & 42 \\
\hline Optimized & 13 & 30 & 45 \\
\hline
\end{tabular}

Figure 3 : Complexity of next state function (literal count) for several path lengths

One can state that Radix- 2 code is to be used in the case of a controllers with large paths.

\section{FINITE STATE MACHINE COMPLEXITY}

Commonly the complexity of controllers is related to the number of states and the number of inputs and/or outputs. Other important parameters have a critical influence on the efficiency of the state assignment. One of them is the complexity, in terms of literal counts, of the predicates on transitions between states. This parameter is correlated with the outdegree of states, i.e the number of transitions origin from the same state. Another topological characteristic of a state machine is the existence of paths. Paths and their lengths and their number of occurrences are very important if a partitioning strategy is adopted particularly in the case of controllers with more than one hundred states (Belhadj, 1993). A particular attention is given to this characteristic. In this paper, two sets of controllers are considered. The first one, called small sequencers, are state machines with a reduced number of states . The second set includes controllers with a number of states greater than 50 .

\section{THE TARGET TECHNOLOGIES}

The experimental study concerns the synthesis of a large set of industrial sequencers using the pervious state assignments on the following technologies : Xilinx, Actel, Crosspoint, QuickLogic and standard cells. Mapping on Max and MACH PLDs is in progress and the whole results will be included in the final version of this paper.

The choice of this set of technologies for mapping is motivated by the significant variety of their characteristics. Some significant differences and their influences are discussed.

i) The FPGAs have a larger number of registers than CPLDs ; but have finer logic granularity

ii) The fanin and fanout of basic modules are typically small for FPGAs than for CPLDs

iii) The FPGA architecture requires incremental extra routing resources to send external and internal signals to each of its loads; whereas in CPLD all the bank of registers within a block 
are accessible to its inputs without need of extra resources. Some CPLDs architecture has shared and parallel expanders that provide additional product terms directly to a macro cell (MAX7000 from ALTERA). The use of these expanders ensures that the logic is synthesized with the fewest logic resources to obtain the fastest possible speed.

The standard cell and PLAs technologies are not addressed here since the most important state assignments were specifically developed for these technologies. The crosspoint technology is closed to standard cells and the results should be closed or similar.

A first consequence is that the FPGAs require next state and output functions that fan out as little as possible. Another consequence is that it is necessary to reduce the number of state variables for implementation on CPLDs, since the reduction of state variables increases the combinational logic parts of the state machine, and allows a higher use of their logic blocks .

According to these remarks, we could predict that the optimized state assignment, Gray and sequential codes are more appropriate than the one hot code for implementations on CPLDs. The one hot encoding should be suitable for a mapping on FPGAs due to the availability of large number of registers and the fine granularity of their architectures.

The optimization criterion (speed, area, trade offs) has a considerable influence. It is expected that the one hot encoding leads to the best critical paths if the delay is the criterion fixed by the user. But this statement must be taken with care, especially when the number of states of the controller is reduced (less than 15). To explain this, let us assume that $\mathrm{N}$, the number of states, is reduced and that it will be implemented on Xilinx. Then N CLBs are needed for the one hot code and $[\log 2(\mathrm{~N})]$ CLBs are necessary for the other encoding techniques. Even if the major parts of logic blocks of these N CLBs are not used the critical path increases due to extra-delays introduced by intra-CLBs connections (see i) and ii)).

If the optimisation criterion is area, the choice of the encoding technique becomes more complex. It considers all the parameters introduced.

\section{EXPERIMENTAL RESULTS}

All the benchmarks have been run first using the ASYL area option then the speed option with the compact, random, sequential, one hot and Gray codes for implementation on standardcell, FPGAs (Xilinx 4000, Actel (ACT2), QuickLogic, Crosspoint) and MACH. The given area (or number of modules) results are obtained using the area option and the criticalpath results are obtained using the speed option.
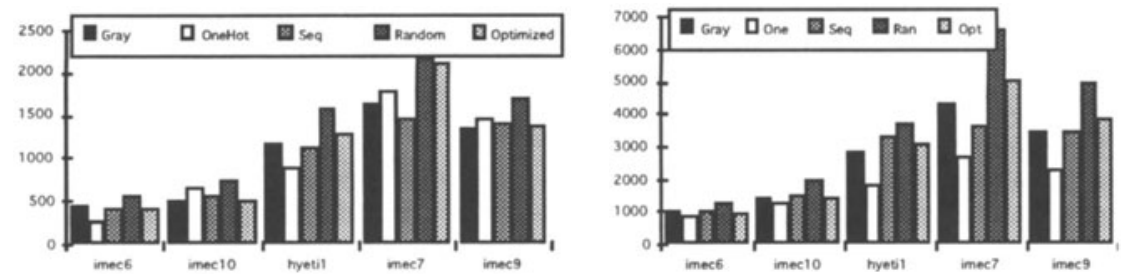

Figure 4: Area measures for ACT2. Figure 5: Area results for standard. cells (VSC370).

Figures 4 and 5 give the area measures for the complex controllers implemented on respectively Actel (ACT2) and standard cells (VSC370 library). Finally, Figures 6 and 7 show the area obtained for AMD PAL22V10 and MACH. 


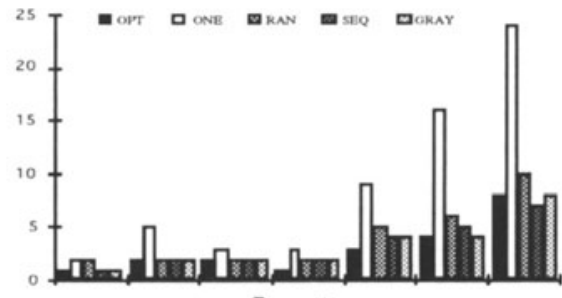

Figure 6: Nb of blocks for AMD PAL22V10.



Figure 7: $\mathrm{Nb}$ of blocks for AMD MACH 130.

\section{Results analysis for the area option}

A quick analysis of the area results shows that the compact encoding leads to the best results for the small sequencers (less than 50 states), with an exception for those that exhibit long paths, for which better results are obtained using linear codes, and this is true for all the targets. For complex sequencers (controllers with more than 50 states), the one hot encoding, as expected, leads to the best results for implementation on standard cells and FPGAs with some exceptions for controllers containing large amount of paths. But as we stated the one hot encoding is not suitable for PLDs. For these technologies, the state assignments with the minimum number of internal variables are more suitable. The compact, sequential and Gray options give approximatively the same results depending on the complexity of the predicates on the transitions and the existence or not of long paths. Table II summarizes these conclusions and draws a rought version of the state assignment selection strategy when the performance criterion is the area.

\begin{tabular}{|c|c|c|c|c|c|}
\hline & $2<\#$ states $<15$ & $15<\#$ states $<50$ & $15<\#$ states $<50$ & \#states $>50 * *$ & \#states $>50$ \\
\hline & & Long Paths & Short Paths & Long Paths & Short Paths \\
\hline Std Cells & Compact & Seq/Gray & Compact & OneHot/Comp. & One Hot \\
\hline Xilinx & Compact & Seq/One Hot & Comp./One Hot & One Hot/Seq & One Hot \\
\hline Actel & Compact & Seq/Gray & Compact & Seq/OneHot & One Hot \\
\hline QuickLogic & Compact & Seq/Gray & Compact & Seq/OneHot & One Hot \\
\hline Crosspoint & Compact & Gray/Seq & Compact & One Hot/Seq & One Hot \\
\hline PAL & Compact & Seq/Gray & Compact & Seq/Gray & Compact \\
\hline MACH & Compact & Compact/Seq & Compact & Seq/Gray & Compact \\
\hline
\end{tabular}

Table II: State assignment selection strategy for area criterion.

\section{Results analysis for the speed option}

The analysis of the delays obtained with the ASYL speed option confirms the expectation that the one hot encoding is globally suitable for all the targets with some exception where the radix-2 (sequential) option is better due to the existence of long paths. However, this is not the case for very small controller (with less than 15 states), for which the state assignement options with the minimum number of state variables lead to more valuable critical paths. Thus the experimental results validate the statements informally introduced in the previous sections. The Table III summarizes these remarks and draws the state assignment selection strategy when the delay is the performance criteria. 


\begin{tabular}{|l|c|c|c|c|c|}
\hline & $2<\#$ states $<15$ & $15<\#$ states $<50$ & $15<\#$ states $<50$ & \#states $>50$ & \#states $>50$ \\
\hline & & Long Paths & Short Paths & Long Paths & Short Paths \\
\hline Std Cells & Seq./Comp. & Seq./One Hot & One Hot & One Hot/Seq. & One Hot \\
\hline Xilinx & Seq./Comp. & One Hot & One Hot & One Hot & One Hot \\
\hline Actel & Seq./Comp. & One Hot & One Hot & One Hot/Seq. & One Hot \\
\hline QuickLogic & Seq/Comp. & One Hot & One Hot & One Hot/Seq. & One Hot \\
\hline Crosspoint & Seq./Comp. & One Hot & One Hot & One Hot/Seq. & One Hot \\
\hline PAL & Seq./Comp. & Seq./One Hot & One Hot & Seq./One Hot & One Hot \\
\hline MACH & Seq./Comp. & Seq./One Hot & One Hot & Seq./One Hot & One Hot \\
\hline
\end{tabular}

Table III: State assignment selection strategy for delay criterion.

\section{CONCLUSION}

This paper introduces a strategy for the selection of a state assignment based on a set of parameters that includes the intrinsic complexity of the considered controller, the technological target and the performance criterion fixed by the designer.

Future works will explore several improvements of the optimized state assignment in order to make different versions; each one is more or less specific to a category of controller and a technological target given a fixed performace criterion. An other direction of investigation concerns the comparison between different implementations, within a partitioning strategy, using a ROM or PLAs and logic part (for multiple output branching primitives) implemented on standard cells, on FPGAs or Complex PLDs. The results of these extensionswill be used as basic start point for efficient synthesis of communicating FSMs (Saucier, 1992).

\section{REFERENCES}

R. Amman et al.. (1988) PLA Based Finite State Machines Using Johnson Counters as State Memories. ICCD, 267-270.

H. Belhadj et al. (1992) Specification and Synthesis of Communicating Finite State Machines. Synthesis For Control Dominated Circuits, Saucier and Trilhe Ed. North Holland.

S. Devadas. (1989) General Decomposition of Sequential Machines: Relationship to State Assignment. 26th DAC, 333-338.

S. Devadas et al. (1987) MUSTANG: State Assignment of FSM for Optimal Multi-Level Implementations. ICCAD, 16-19.

S. Devadas and A. R. Newton (1988) Decomposition and Factorisation of Sequential Finite State Machines. ICCAD, pp. 148-151.

G. de Micheli, et al. (1983) Comp. Aided Synthesis of PLA-based FSMs. ICCAD, 154-156.

C. Duff. (1991) Codage d'automates et théorie des cubes intersectants, I.N.P. Grenoble.

S. Golson. (1992) One Hot state machine design for FPGAs. Int'1 Workshop on Logic Synthesis.

L. Gerbaux. G. Saucier. (1992) Automatic Synthesis of Large Moore Sequencers. EDAC.

J. Hartmanis and R.E. Stearns. (1966) Algebraic Structure Theory for Sequential Machines. PrenticeHall, Englewood Cliffs, N. J.

S. Hassoun and G. Boriello. (1993) Improving Finite State Assignment for Two-Level PLDs. International Workshop on Logic Synthesis, Califonia.

Bill Lin and A. R. Newton. (1989) Synthesis for Multiple-Level Logic from Symbolic High Level Description Languages. VLSI' 89 Conference, 187-196, Germany.

P. Paulin. (1989) Horizontal Partitioning of PLA-based Finite State Machines. DAC, 333-338.

T. Villa, et al.. (1986) NOVA : state Assignment of Finite State Machines for Optimal Two Level Logic Implementations.26th DAC. Las Vegas. 